\title{
Numerical Technique for Wireless Communication System with High Speed Movement
}

\author{
Shafrida Sahrani*, Michiko Kuroda ${ }^{\dagger}$ \\ *Faculty of Engineering, Universiti Malaysia Sarawak, Malaysia \\ E-mail: sshafrida@feng.unimas.my \\ †Tokyo University of Technology, Japan \\ E-mail: kuroda@stf.teu.ac.jp
}

\begin{abstract}
Numerical technique for the analysis of EM field with high speed moving dielectric body by using FDTD method with Overset Grid Generation method considering Lorentz transformation is presented. The characteristic of EM field when incident wave hits the moving dielectric body with high velocity value are analyzed. The accuracy of the proposed technique is validated. Good agreements are obtained between numerical results and theoretical results. The development of this numerical technique will give a great impact for many areas, particularly for the future high speed mobile communication systems used in transportation and aerial radar systems to detect the high speed motion of a moving boundary.
\end{abstract}

Keywords-FDTD method; moving boundary problem; Overset Grid Generation method; Lorentz transformation; dielectric body

\section{INTRODUCTION}

The analysis of the electromagnetic (EM) field around a moving body has become an essential tool for the realization of future electromagnetic applications [1]-[3]. A new numerical technique by using FDTD method combined with Overset Grid Generation method considering Lorentz transformation is proposed. The preliminary version of this proposed technique for the case of the moving/rotating body considering the Doppler Effect is already presented in [4],[5].

The Overset Grid Generation method consists of a main mesh and a sub-mesh that overlapped each another [6]. The main mesh covers the entirety of the computational domain while the sub-mesh is used to model the moving body with high velocity value considering Lorentz transformation. Based on the central difference equation in FDTD method, the same space and time increment are required in each calculation [4]. Here, the time components that were changed by Lorentz transformation are fixed by using interpolation scheme in the Overset Grid Generation method. This allows a coherent point in time component with the FDTD method, which is an advantage of our proposed numerical technique.

This paper discusses the transmission and reflection of EM field when the incident wave hits the uniformly moving dielectric body with high velocity value. The analysis is validated by comparing numerical and theoretical results. Good agreements between both results confirm the accuracy of the proposed technique. This will give important insights into the capability of the proposed numerical technique to solve the EM field problem with high speed moving boundaries.

\section{EM FIELD AROUND MOVING BodY}

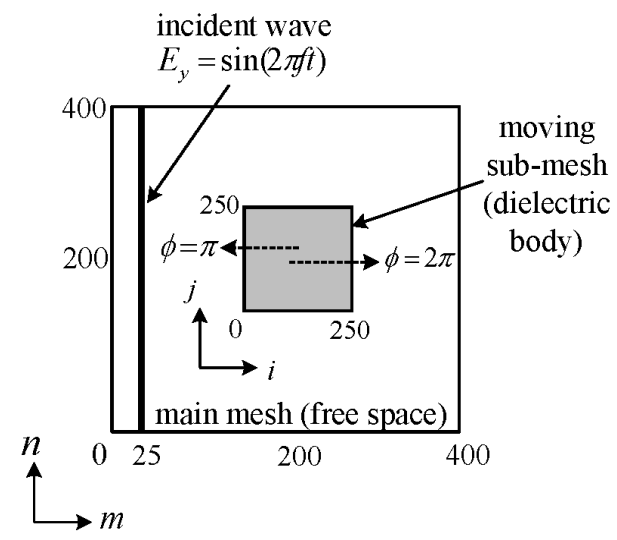

Fig. 1 Numerical model of the moving dielectric body.

Fig. 1 shows the numerical model of the moving dielectric body on the overlapped main mesh. In this analysis, the submesh is assumed to be the dielectric body and the main mesh is in free space. The number of the grids on the main mesh $(m, n)$ is set to be $400 \times 400$ and the number of the grids on the sub-mesh $(i, j)$ is set to $250 \times 250$ grids. The space increment for the main mesh is $\Delta x_{m}=\Delta y_{m}=\lambda / 60$, and the space increment for the sub-mesh is $\Delta x_{s}=\Delta y_{s}=\lambda / 300 . \lambda$ is the wavelength of the incident wave. The meshes are terminated with Mur's absorbing boundary conditions [7].

The time increment, $\Delta t$ is set to $5.0 \times 10^{-14}(\mathrm{~s})$ to meet the stability criterion, $\Delta t \leq \Delta x_{s} / c$, where $c$ is the velocity of the light. The incident wave is $E_{y}=\sin (2 \pi f t)$ where the frequency, $f=100(\mathrm{GHz})$ input at $m=25$ on the main mesh. The sub mesh is set in different dielectric constants, $\varepsilon_{r}=1.5$ and $\varepsilon_{r}=2.5$, respectively in the analysis. The characteristic of EM field around the dielectric body are investigated in stationary case, move towards $(\phi=\pi)$ and against $(\phi=2 \pi)$ the incident wave with velocity, $v=0.1 c(\mathrm{~m} / \mathrm{s})$. The numerical results are shown in Fig. 2 to Fig. 5. 